\title{
A First-Principle Study of B- and P-Doped Silicon Quantum Dots
}

\author{
Jieqiong Zeng and Hong Yu \\ Key Laboratory of MEMS of Ministry of Education, Southeast University, Nanjing 210096, China \\ Correspondence should be addressed to Hong Yu, h_yu@seu.edu.cn
}

Received 24 February 2012; Accepted 26 April 2012

Academic Editor: Sergio J. Mejía-Rosales

Copyright (C) 2012 J. Zeng and H. Yu. This is an open access article distributed under the Creative Commons Attribution License, which permits unrestricted use, distribution, and reproduction in any medium, provided the original work is properly cited.

\begin{abstract}
Doping of silicon quantum dots (Si QDs) is important for realizing the potential applications of Si QDs in the fields of Si QDsbased all-Si tandem solar cells, thin-film transistors, and optoelectronic devices. Based on the first-principle calculations, structural and electronic properties of hydrogen terminated Si QDs doped with single Boron (B) or phosphorus (P) are investigated. It is found out that the structural distortion induced by impurity doping is related to the impurity characteristic, impurity position, and the QD size according to the structural analysis. The relative energetic stability of Si QDs with a single impurity in different locations has been discussed, too Furthermore, our calculations of the band structure and electronic densities of state (DOS) associated with the considered Si QDs show that impurity doping will introduce impurity states within the energy gap, and spin split occurs for some configurations. A detailed analysis of the influences of impurity position and QD size on the impurity levels has been made, too.
\end{abstract}

\section{Introduction}

As we all know, the ultimate reduction in the dimensionality of a semiconductor device will result in a quantum dot. Much attention has been drawn to Si QDs, with promising applications in fields such as optoelectronics [1], photovoltaics [2], and data storage [3]. In order to realize these potential applications of Si QDs, it is essential to develop techniques to control the electronic and optical properties of Si QDs. One such technique currently being investigated is impurity doping $[4,5]$. Doping of Si QDs is expected to introduce additional levels close to the HOMO (highest occupied molecular orbital) or LUMO (lowest unoccupied molecular orbital) in the same fashion as it does for bulk Si; for instance, a nanoscaled P-N junction formed by doping Si QDs embedded in a dielectric is proposed as a way to dissociate photogenerated electron-hole pairs [6].

Lots of experimental works have been made to study the electronic and optical properties of doped Si QDs. However, several factors contribute to making these studies difficult tasks. For instance, QDs synthesized by using different techniques often show different properties in size, shape, and in the interface structure. Moreover, it is tremendously challenging to control over impurity concentration and precise positioning by experiments. This leads to the imperative need for theoretical calculations to predict the properties of doped Si QDs. Compared with calculations for pure, undoped systems, calculations for doped Si QDs are much more complicated and time consuming owing to the variety of atoms and low symmetry. To date, only few first-principle studies devoted to doped Si QDs are presented in the literature. Melnikov and Chelikowsky [7] investigated the electronic properties of hydrogenated Si QDs doped with a single phosphorus atom using a real-space ab initio pseudopotential method, and the ionization energy and binding energy of the defect were calculated. Cantele et al. [8] reported on a detailed first-principle calculation of the impurity states in B- and P-doped Si QDs; the formation energies for neutral impurities were discussed. Although the size effect on the impurity levels were often studied in the previous work, the influences of different impurity positions on the band structures and DOSs of doped Si QDs were not very often examined.

In this paper, we carry out first-principle calculations of impurity states in spherical Si QDs doped with single B or $\mathrm{P}$ atom. Both the structural and electronic properties (band structure and DOS) are investigated as functions of the QD size and of the impurity position within the Si QD. Since it has been demonstrated that the properties of doped $\mathrm{Si}$ QDs critically depend on the location of the impurity atom 
$[9,10]$, the relative energetic stability of Si QDs with a single impurity in all the possible locations has been discussed. Owing to the highly demanding computational costs, we limit our calculations to Si QDs with two different sizes; one is $\mathrm{Si}_{35} \mathrm{H}_{36}$-based QD (approximately $1.1 \mathrm{~nm}$ in diameter), and the other is $\mathrm{Si}_{87} \mathrm{H}_{76}$-based QD (approximately $1.6 \mathrm{~nm}$ in diameter). The $\mathrm{B}$ or $\mathrm{P}$ impurity is considered in substitution site, which is the most stable site for these two impurities [11].

\section{Modeling and Calculation}

Our first-principle calculations are performed by the CASTEP software package [12] which employs the planewave pseudopotentials method based on the density functional theory (DFT). The generalized gradient approximation (GGA) with ultrasoft pseudopotentials in reciprocal space is adopted, and exchange-correlation potential is parametrized by the Perdew-Burke-Ernzerhof scheme (PBE) [13]. Since for a single B- or P-doping, the doped QDs have an odd number of electrons, spin polarization is included in our calculations. The cutoff energy for the wave functions is $190 \mathrm{eV}$. It has been shown that the results are well converged at this cutoff energy. The SCF tolerance is $2.0 e-6 \mathrm{eV} /$ atom, and the $\mathrm{k}$-points for the Brillouin-zone sampling are set as Gamma point only in all cases.

As mentioned above, we consider Si QDs with two different sizes here; one is $\mathrm{Si}_{35} \mathrm{H}_{36}$-based QD (approximately $1.1 \mathrm{~nm}$ in diameter), and the other is $\mathrm{Si}_{87} \mathrm{H}_{76}$-based QD (approximately $1.6 \mathrm{~nm}$ in diameter). Initial undoped dots have been obtained by taking all the bulk Si atoms contained within a sphere of a given radius and passivating the surface dangling bonds with hydrogen. For doped dots, we use the optimized undoped geometry as a base and substitute a $\mathrm{Si}$ atom with an impurity atom. A simple-cubic supercell of vacuum is built for each considered dot which is placed at the center in order to prevent interactions between the periodic replicas. In this work we choose $30 \AA$ and $35 \AA$ as the supercell side lengths for $\mathrm{Si}_{35} \mathrm{H}_{36}$-based QDs and $\mathrm{Si}_{87} \mathrm{H}_{76}$-based QDs, respectively.

Since the details of the QD surface are different for QDs synthesized by using different techniques, here the impurity atom is not allowed to replace an $\mathrm{Si}$ atom on the surface of the QD that is passivated by one or two $\mathrm{H}$ atoms. For the remaining sites, there are two different sites within the QD for a single substitution impurity in the case of $\mathrm{Si}_{35} \mathrm{H}_{36}$, whereas in the case of $\mathrm{Si}_{87} \mathrm{H}_{76}$ there are five. These different sites within the dots are shown in Figure 1. All these geometries are examined for impurity doping to investigate the effects of the impurity position. Here we assign $\mathrm{Si}_{34} \mathrm{XH}_{36}-1, \mathrm{Si}_{34} \mathrm{XH}_{36}-2$, and $\mathrm{Si}_{86} \mathrm{XH}_{76}-1, \mathrm{Si}_{86} \mathrm{XH}_{76}-2$, $\mathrm{Si}_{86} \mathrm{XH}_{76^{-}} 3, \mathrm{Si}_{86} \mathrm{XH}_{76^{-}}, \mathrm{Si}_{86} \mathrm{XH}_{76}-5$ (X represents $\mathrm{B}$ or $\mathrm{P}$ ) corresponding to the doped Si QD whose impurity atom situates in the site marked by 1, 2 in Figure 1(a), and 1, 2, 3, 4, 5 in Figure 1(b), respectively.

For each model, the first step is to relax the structure with all the freedom. Once the optimization is achieved, the related properties are calculated. However, even accurate

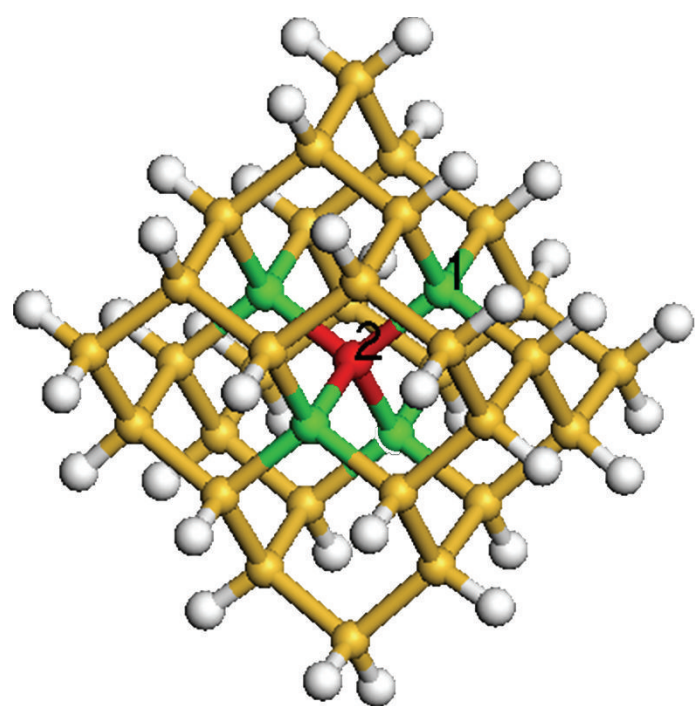

(a)

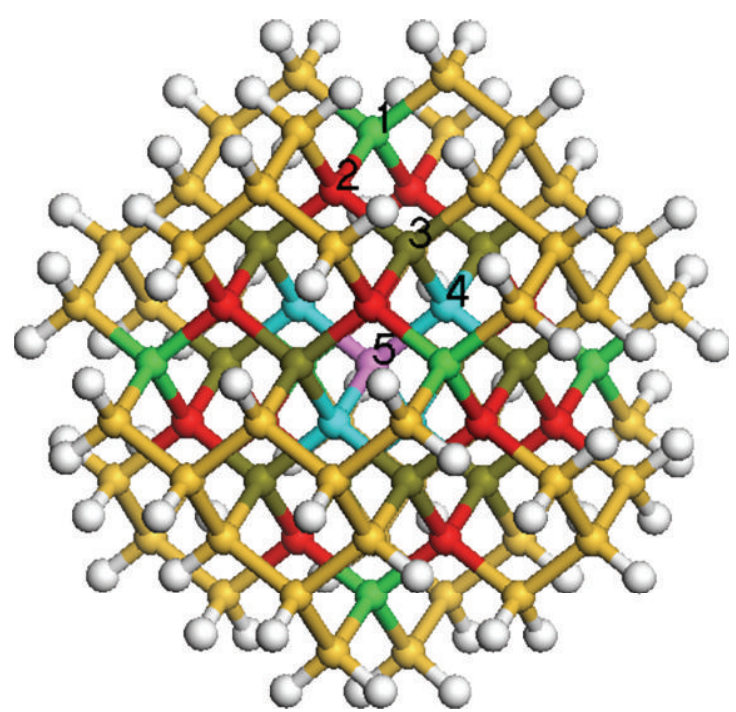

(b)

FIGURE 1: A schematic representation of different sites within the QDs for a single substitution impurity. $\mathrm{H}$ and $\mathrm{Si}$ atoms are denoted by white and yellow balls, respectively. The sites shaded by a same color within the QDs correspond to equivalent doping sites. (a) Model of a $1.1 \mathrm{~nm} \mathrm{Si} \mathrm{QD}\left(\mathrm{Si}_{35} \mathrm{H}_{36}\right)$; the two different sites within the QD are marked by 1 and 2; (b) model of a $1.6 \mathrm{~nm} \mathrm{Si} \mathrm{QD}\left(\mathrm{Si}_{87} \mathrm{H}_{76}\right)$; the five different sites within the QD are marked by 1, 2, 3, 4, and 5 .

ab initio calculations performed within GGA will suffer from the well-known underestimation of the energy gap. Here we leave the underestimation of energy gap uncorrected and do not discuss the absolute value of the energy gap but focus on how the band structure and DOS are affected by a single impurity doping. Thus it can provide some guidance to properly utilizing impurity doping to control the electronic and optical properties of Si QDs. 

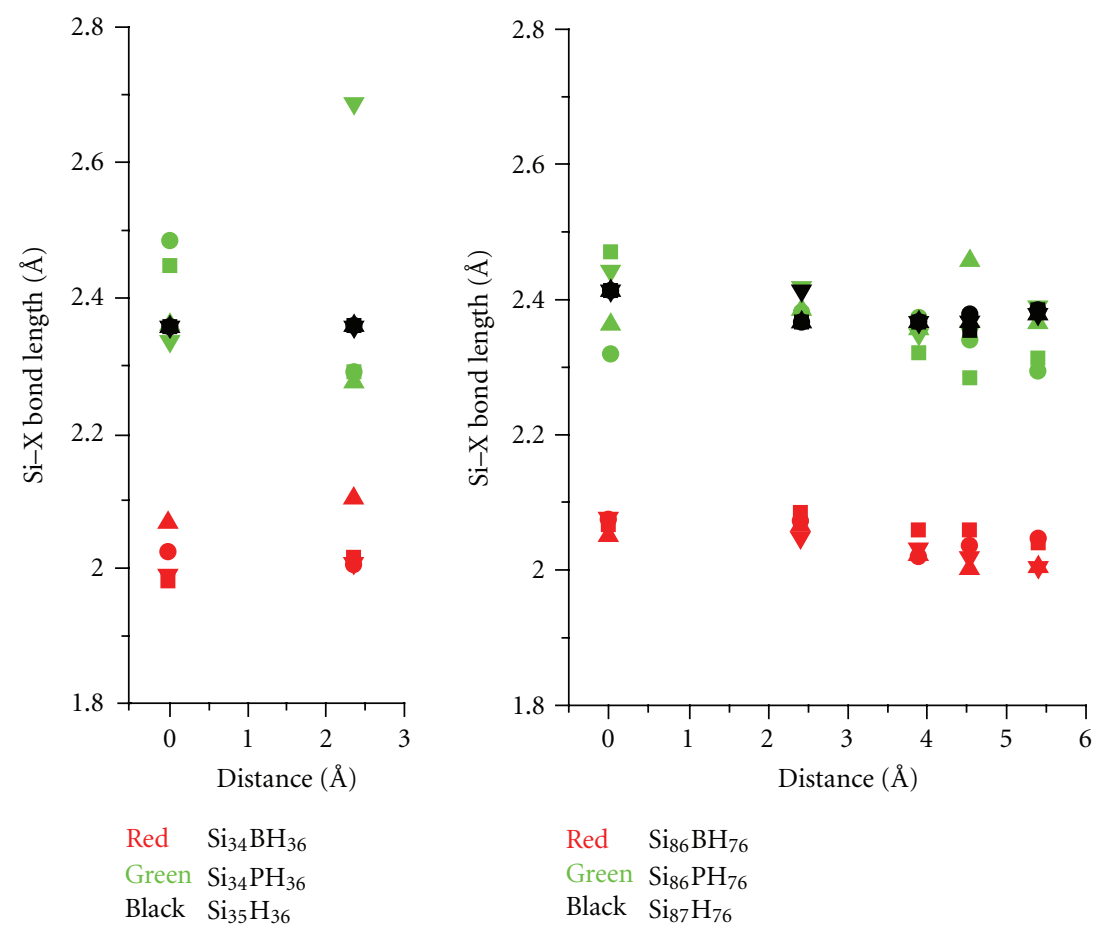

FIGURE 2: The optimized bond lengths around the impurity site for the undoped Si QDs (black ones, X = Si), B-doped Si QDs (red ones, $\mathrm{X}=\mathrm{B}$ ), and P-doped Si QDs (green ones, $\mathrm{X}=\mathrm{P}$ ). The $x$-axis measures the distance from the center to the replaced Si atom in undoped QDs. The four different Si-X bond lengths are indicated by $\mathbf{Q}, \mathbf{\square}, \boldsymbol{\Delta}$, and $\boldsymbol{\nabla}$, respectively.

\section{Results and Discussion}

3.1. Structural Properties. The structural changes induced by the presence of a single impurity with respect to the corresponding undoped ones have been investigated. It is observed that with the insertion of one impurity, some variations in the bond lengths occur around the impurity site, whereas the $\mathrm{Si}-\mathrm{Si}$ bond lengths remain almost unchanged. Figure 2 shows the optimized bond lengths around the impurity site for the doped and undoped Si QDs. Our results for the bond lengths are in very good agreement with the results reported by Iori et al. [14]. From Figure 2 it can be seen that, compared with undoped cases in which the four Si-Si bond lengths are practically the same, there is a general trend to a significant decrease of the bond lengths for B-doped Si QDs. However, for P-doped cases there exists expansion of the bond lengths with respect to the undoped cases, and the changes are less than B-doped cases. Moreover, for Pdoped cases the structural distortion is strongly dependent on the QD size; the larger the size, the less the distortion, while for B-doped cases the distortion is nearly independent of the size. In addition, as is shown in Figure 2, the relaxation around the impurity is also related to the impurity position. By comparing the $\mathrm{Si}_{35} \mathrm{H}_{36}$-based cases and $\mathrm{Si}_{87} \mathrm{H}_{76}$-based cases, we find out that with the decrease of the QD size the impact of impurity position on the structure relaxation becomes more significant.

From the discussion above, we know that the configurations for single doped Si QDs are different from each other due to different impurity positions. So it is needed to explore which configuration is the most stable one. The relative energetic stability of these Si QDs can be evaluated through the calculation of the formation energy (FE). Starting from the $\mathrm{Si}_{n} \mathrm{H}_{m}$ nanocluster, the FE for the neutral X impurity can be depicted in (1) [8]:

$$
\mathrm{FE}=E\left(\mathrm{Si}_{n-1} \mathrm{XH}_{m}\right)-E\left(\mathrm{Si}_{n} \mathrm{H}_{m}\right)+\mu_{\mathrm{Si}}-\mu_{\mathrm{X}},
$$

where $E$ is the total energy of the system, $\mu_{\mathrm{Si}}$ is the total energy per atom of bulk $\mathrm{Si}$, and $\mu_{\mathrm{X}}$ is the total energy per atom of the impurity. From (1), we find out that the relative value of the FE for doped Si QDs with all kinds of B (or P) configurations does not depend on $\mu_{\mathrm{Si}}$ and $\mu_{\mathrm{B}}$ (or $\left.\mu_{\mathrm{P}}\right)$. It is proportional to the difference between $E\left(\mathrm{Si}_{n-1} \mathrm{XH}_{m}\right)$ and $E\left(\mathrm{Si}_{n} \mathrm{H}_{m}\right)$. Thus, the relative stability may be determined by simply comparing the differences of total energies between doped QDs and corresponding undoped ones.

The calculated differences of total energies as functions of the impurity position are shown in Figure 3. We observe that the energies required for impurity $\mathrm{X}(\mathrm{X}=\mathrm{B}, \mathrm{P})$ doping into Si QDs increase in the order $\mathrm{Si}_{34} \mathrm{XH}_{36}-1<\mathrm{Si}_{34} \mathrm{XH}_{36}-2$ for $\mathrm{Si}_{35} \mathrm{H}_{36}$-based QDs. While in the case of $\mathrm{Si}_{87} \mathrm{H}_{76}$-based QDs, for B-doping the order is $\mathrm{Si}_{86} \mathrm{BH}_{76}-2<\mathrm{Si}_{86} \mathrm{BH}_{76}-4<$ $\mathrm{Si}_{86} \mathrm{BH}_{76}-5<\mathrm{Si}_{86} \mathrm{BH}_{76}-1<\mathrm{Si}_{86} \mathrm{BH}_{76}-3$, and for P-doping the order is $\mathrm{Si}_{86} \mathrm{PH}_{76^{-}}-<\mathrm{Si}_{86} \mathrm{PH}_{76^{-}}-<\mathrm{Si}_{86} \mathrm{PH}_{76^{-}} 1<\mathrm{Si}_{86} \mathrm{PH}_{76^{-}}$ $4<\mathrm{Si}_{86} \mathrm{PH}_{76}-3$. This means that $\mathrm{Si}_{34} \mathrm{XH}_{36}-1\left(\mathrm{Si}_{86} \mathrm{XH}_{76}-2\right)$ is energetically the most favorable configuration for either B- or P-doping in $\mathrm{Si}_{35} \mathrm{H}_{36}\left(\mathrm{Si}_{87} \mathrm{H}_{76}\right)$ owing to the smallest formation energy. So we find out that for either B- or Pdoping, the impurity atom tends to substitute $\mathrm{Si}$ atom near 


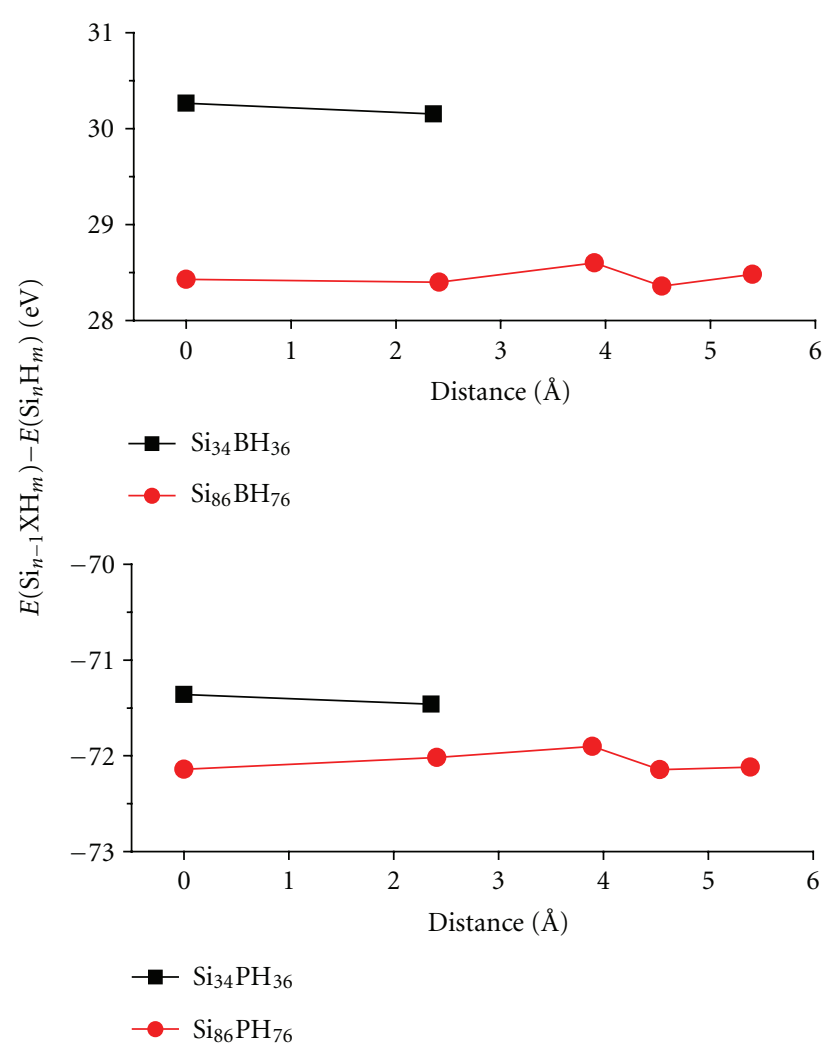

Figure 3: Differences of total energies between doped QDs with different impurity positions and corresponding undoped ones. The $x$-axis measures the distance from the center to the replaced Si atom in undoped QDs. Different $x$ value corresponds different impurity position. Along the increasing direction of $x$-axis, they are in the order of $\mathrm{Si}_{34} \mathrm{XH}_{36}-2, \mathrm{Si}_{34} \mathrm{XH}_{36}-1$ for $\mathrm{Si}_{35} \mathrm{H}_{36}$-based QDs, and for $\mathrm{Si}_{87} \mathrm{H}_{76}$-based QDs the order is $\mathrm{Si}_{86} \mathrm{XH}_{76}-5, \mathrm{Si}_{86} \mathrm{XH}_{76}-4, \mathrm{Si}_{86} \mathrm{XH}_{76^{-}}$ $3, \mathrm{Si}_{86} \mathrm{XH}_{76}-2, \mathrm{Si}_{86} \mathrm{XH}_{76}-1$.

the surface. Moreover, it can be observed that the energy required for impurity $\mathrm{X}(\mathrm{X}=\mathrm{B}, \mathrm{P})$ doping is smaller for $\mathrm{Si}_{87} \mathrm{H}_{76}$ than for $\mathrm{Si}_{35} \mathrm{H}_{36}$, demonstrating that with a decrease of the QD size the insertion of a substitution impurity becomes more difficult. In addition, we find out that this influence of size is much more evident for B-doping than for P-doping (see Figure 3), meaning that it would be even harder to conduct B-doping for Si QD with smaller size.

3.2. Electronic Properties. The calculated energy levels for doped and undoped Si QDs are depicted in Figure 4. As can be seen, the B-doping gives rise to impurity states located above the valence band, whereas the P-doping gives rise to impurity states located below the conduction band. These impurity-related levels falling within the gap are strongly focused either on B or P impurity atom, as one can see from Figure 5 where the localization of these impurity states is shown.

One can see from Figure 4 that owing to the impurity doping, spin split occurs for some doped QD configurations. It is obvious that the energy differences between spin-up and spin-down levels in smaller doped Si QDs are more pronounced than the ones in the bigger doped QDs case. Besides, for QDs with the same size but different impurity positions, the situations of spin split are different from each other, particularly for $\mathrm{Si}_{87} \mathrm{H}_{76}$-based QDs in which split disappears in several configurations. So spin split induced by doping depends on not only the QD size but also the impurity position. In addition, it is observed that the impurity position also has an impact on the position of impurity levels. For $\mathrm{Si}_{35} \mathrm{H}_{36}$-based QDs, as the impurity moves from the QD subsurface toward the center, the impurity states become deeper (shallower) for B-doping (Pdoping). However, in the case of $\mathrm{Si}_{87} \mathrm{H}_{76}$-based QDs, as the impurity moves from the QD subsurface toward the center, the impurity states become shallower (deeper) for B-doping (P-doping). This indicates that the impurity position has opposite effects on the impurity levels for B-doping and for P-doping, and the ultimate effects also closely depend on the QD size. Furthermore, we find out that although B (or P) introduces shallow acceptor (or donor) states in bulk silicon, either B- or P-doping introduces deep energy levels in the energy gap of Si QD. By comparing the impurity levels of the QDs with two different sizes, it can be easily found out that with a decrease of the QD size the acceptor (B-doping) and donor (P-doping) levels become even deeper.

The electronic densities of states (DOSs) for the investigated Si QDs have also been calculated to get a better understanding of the effect of impurity doping on the electronic properties of Si QDs. The DOSs shown in Figure 6 for the B- and P-doped Si QDs are compared with the ones of corresponding undoped Si QDs. As can be seen, the peaks in the DOSs for either B- or P-doped Si QDs show a similar structure with that of the undoped cases out of the energy range of the HOMO-LUMO gap. However, some impurity-related peaks appear just above the HOMO of the undoped QD for B-doped cases, while for P-doped cases the peaks located just below the LUMO of the undoped QD. In addition, it can be seen from Figure 6 that for $\mathrm{Si}_{35} \mathrm{H}_{36}$ based QDs, as the impurity moves from the QD subsurface toward the center, the impurity-related peaks move toward the midgap region (LUMO) for B-doping (P-doping). While for $\mathrm{Si}_{87} \mathrm{H}_{76}$-based QDs, as the impurity moves from the QD subsurface toward the center, the impurity-related peaks move toward the HOMO (midgap region) for B-doping (Pdoping). Moreover, it is obvious that with a decrease of the QD size the impurity-related peaks are closer to the midgap region. All these characteristics show a good agreement with the ones of energy levels discussed before.

\section{Conclusion}

The structural and electronic properties of B- and P-doped Si QDs are investigated by means of first-principle calculations. All the possible locations for a single substitution impurity inside Si QDs are considered. According to the structural analysis, it is found out that for P-doped case the structural distortion is less significant than that for B-doped case. The distortion is strongly dependent on the QD size for P-doped case; the larger the size, the less the distortion. While for 


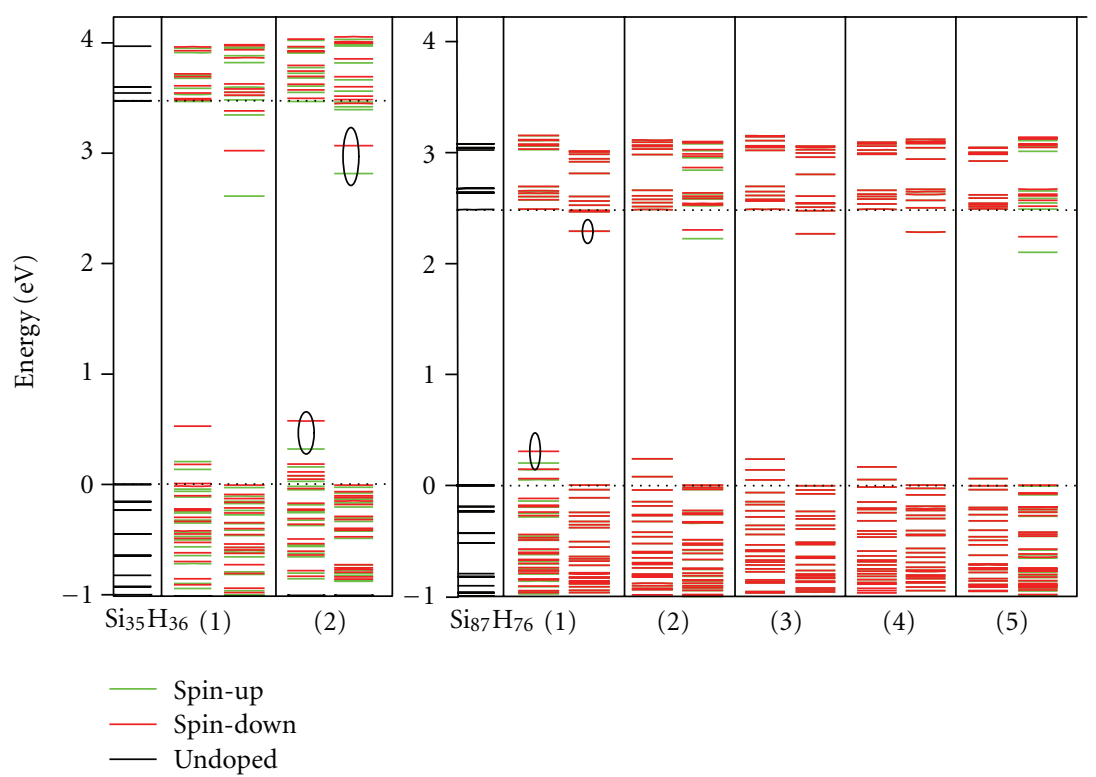

FIGURE 4: Energy level diagram for doped (red and green) and undoped (black) Si QDs. From left to right it is arranged in the order $\mathrm{Si}_{35} \mathrm{H}_{36}$, $\mathrm{Si}_{34} \mathrm{BH}_{36}-1, \mathrm{Si}_{34} \mathrm{PH}_{36}-1, \mathrm{Si}_{34} \mathrm{BH}_{36}-2, \mathrm{Si}_{34} \mathrm{PH}_{36}-2, \mathrm{Si}_{87} \mathrm{H}_{76}, \mathrm{Si}_{86} \mathrm{BH}_{76}-1, \mathrm{Si}_{86} \mathrm{PH}_{76}-1, \mathrm{Si}_{86} \mathrm{BH}_{76}-2, \mathrm{Si}_{86} \mathrm{PH}_{76}-2, \mathrm{Si}_{86} \mathrm{BH}_{76}-3, \mathrm{Si}_{86} \mathrm{PH}_{76}-3, \mathrm{Si}_{86} \mathrm{BH}_{76}-4$, $\mathrm{Si}_{86} \mathrm{PH}_{76}-4, \mathrm{Si}_{86} \mathrm{BH}_{76}-5, \mathrm{Si}_{86} \mathrm{PH}_{76}-5$. Spin-up (spin down) states of doped Si QDs are indicated by green (red). If spin-up state and spin-down state overlap, the overlap turns out to be red. So if the energy levels are all red, it means that there does not exist spin split; otherwise it means that spin split occurs. The dotted lines represent the HOMO and LUMO states of the undoped QD.

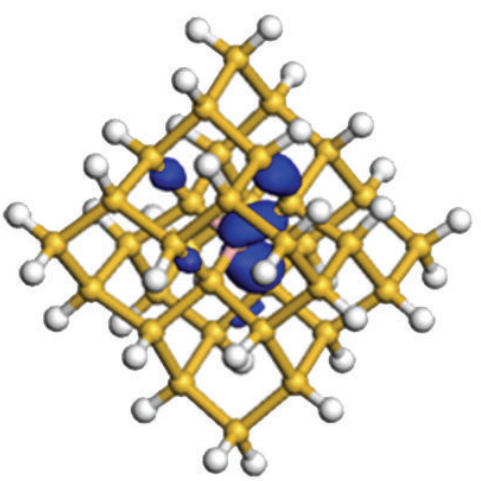

$\mathrm{Si}_{34} \mathrm{BH}_{36}-2$

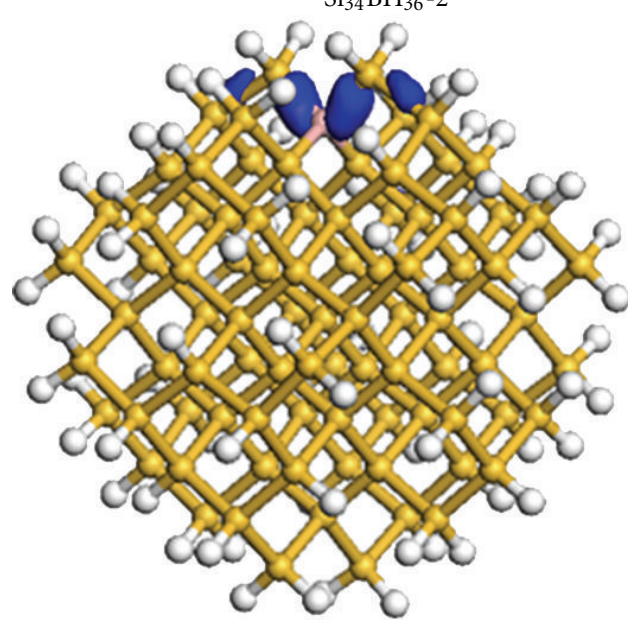

$\mathrm{Si}_{86} \mathrm{BH}_{76}-1$

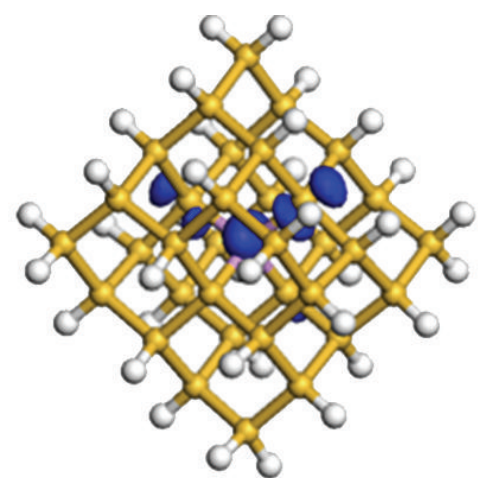

$\mathrm{Si}_{34} \mathrm{PH}_{36}-2$

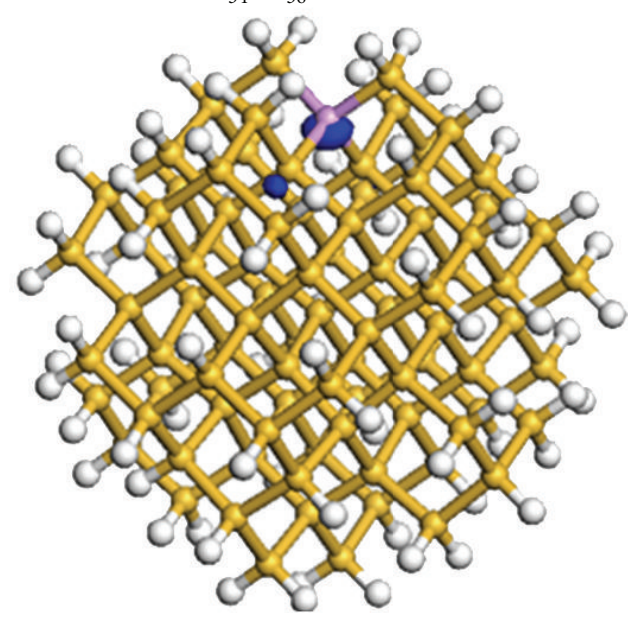

$\mathrm{Si}_{86} \mathrm{PH}_{76}-1$

FIGURE 5: The orbital analysis of defect states (denoted by circles in Figure 4) for doped Si QDs. The isovalue is set as 0.02 for each case. 

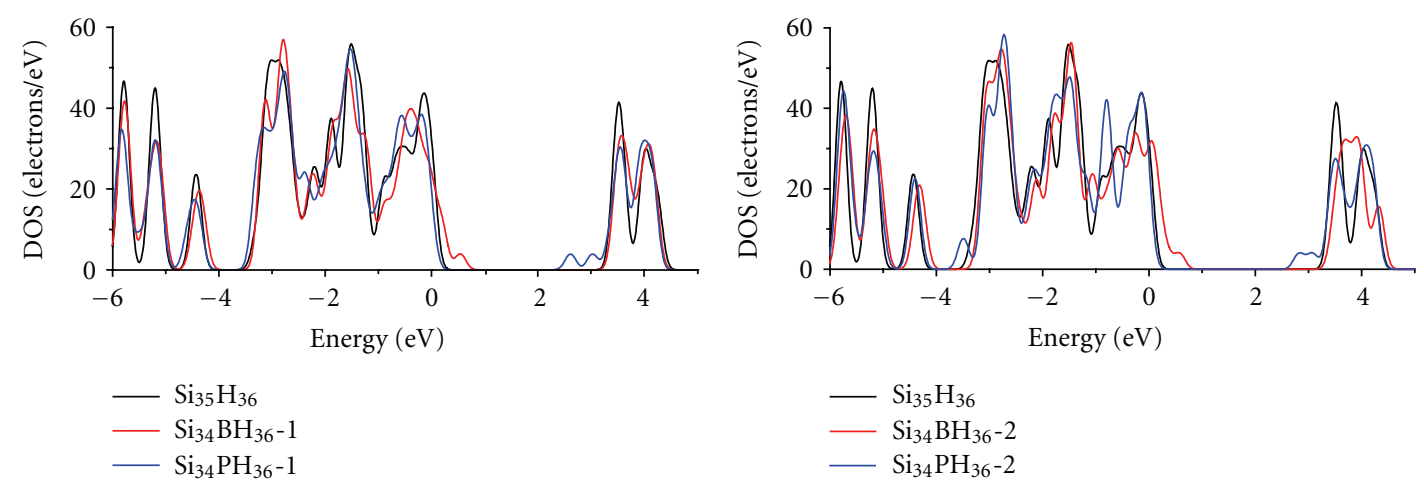

(a)
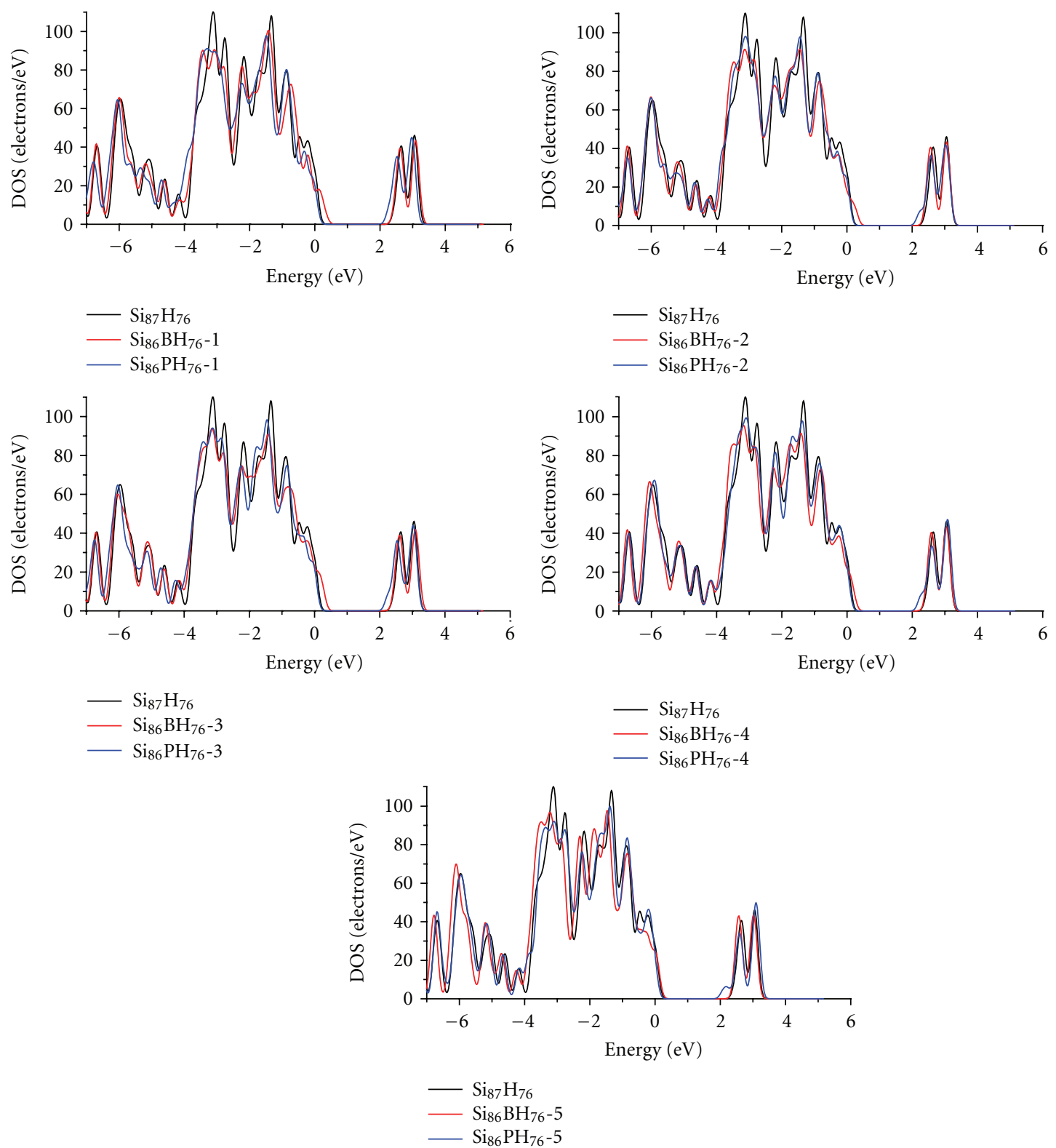

(b)

FIGURE 6: Electronic density of states for undoped Si QDs (black), B-doped Si QDs (red), and P-doped Si QDs (blue). From left to right, from top to bottom, the impurity position moves from the QD subsurface toward the center. (a) The DOSs for $\mathrm{Si}_{35} \mathrm{H}_{36}$-based QDs; (b) the DOSs for $\mathrm{Si}_{87} \mathrm{H}_{76}$-based QDs. 
B-doped case the distortion is nearly independent of the size. The relaxation around the impurity is also related to the impurity position, and the impact of impurity position becomes more significant with a decrease of the QD size. The most stable configuration is explored in all these single doped configurations by the energy calculations. The results show that for either B- or P-doping, the impurity atom tends to substitute $\mathrm{Si}$ atom near the surface. Besides, we find out that with a decrease of the QD size the insertion of a substitution impurity becomes more difficult, and this size effect is much more evident for B-doping than P-doping. In addition, our calculations of the band structure and DOS associated with the considered Si QDs show that the presence of $\mathrm{P}$ atom (or B atom) introduces donor (or acceptor) states within the energy gap, and spin split occurs for some doped QD configurations. Moreover, the influence of impurity position on the impurity levels has been discussed. It is indicated that the impurity position has opposite effects on the impurity levels for B-doping and for P-doping. Furthermore, it is demonstrated that either B- or P-doping introduces deep energy levels in the energy gap of Si QDs, and with a decrease of the QD size the acceptor (B-doping) and donor (P-doping) levels become even deeper.

These insights gained in our study show the possibility of an impurity-based engineering of the Si QD electronic properties, which are of great help to the realization of related Si QD-based potential applications.

\section{Acknowledgments}

This project is partially supported by the National Science Foundation of China (Grant no. 61001044). The authors are grateful to Shanghai Supercomputer Center for providing computation resources.

\section{References}

[1] M. Cazzanelli, D. Navarro-Urriós, F. Riboli et al., "Optical gain in monodispersed silicon nanocrystals," Journal of Applied Physics, vol. 96, no. 6, pp. 3164-3171, 2004.

[2] E. C. Cho, M. A. Green, G. Conibeer et al., "Silicon quantum dots in a dielectric matrix for all-silicon tandem solar cells," Advances in OptoElectronics, vol. 2007, Article ID 69578, 11 pages, 2007.

[3] Z. J. Horváth, "Semiconductor nanocrystals in dielectrics: optoelectronic and memory applications of related siliconbased MIS devices," Current Applied Physics, vol. 6, no. 2, pp. 145-148, 2006.

[4] E. Srinivasan and G. N. Parsons, "Hydrogen elimination and phase transitions in pulsed-gas plasma deposition of amorphous and microcrystalline silicon," Journal of Applied Physics, vol. 81, no. 6, pp. 2847-2855, 1997.

[5] E. A. G. Hamers, A. Fontcuberta I Morral, C. Niikura, R. Brenot, and P. Roca I Cabarrocas, "Contribution of ions to the growth of amorphous, polymorphous, and microcrystalline silicon thin films," Journal of Applied Physics, vol. 88, no. 6, pp. 3674-3688, 2000.

[6] X. J. Hao, E. C. Cho, C. Flynn, Y. S. Shen, G. Conibeer, and M. A. Green, "Effects of boron doping on the structural and optical properties of silicon nanocrystals in a silicon dioxide matrix," Nanotechnology, vol. 19, no. 42, Article ID 424019, 2008.

[7] D. V. Melnikov and J. R. Chelikowsky, "Quantum confinement in phosphorus-doped silicon nanocrystals," Physical Review Letters, vol. 92, no. 4, pp. 468021-468024, 2004.

[8] G. Cantele, E. Degoli, E. Luppi et al., "First-principles study of n- and p-doped silicon nanoclusters," Physical Review B, vol. 72, no. 11, Article ID 113303, 4 pages, 2005.

[9] X. Pi, X. Chen, and D. Yang, "First-principles study of 2.2 $\mathrm{nm}$ silicon nanocrystals doped with boron," Journal of Physical Chemistry C, vol. 115, no. 20, pp. 9838-9843, 2011.

[10] X. Chen, X. Pi, and D. Yang, "Critical role of dopant location for P-doped Si nanocrystals," Journal of Physical Chemistry C, vol. 115, no. 3, pp. 661-666, 2011.

[11] F. Iori, E. Degoli, R. Magri et al., "Engineering silicon nanocrystals: theoretical study of the effect of codoping with boron and phosphorus," Physical Review B, vol. 76, no. 8, Article ID 085302, 14 pages, 2007.

[12] M. D. Segall, P. J. D. Lindan, M. J. Probert et al., "Firstprinciples simulation: ideas, illustrations and the CASTEP code," Journal of Physics Condensed Matter, vol. 14, no. 11, pp. 2717-2744, 2002.

[13] J. P. Perdew, K. Burke, and M. Ernzerhof, "Generalized gradient approximation made simple," Physical Review Letters, vol. 77, no. 18, pp. 3865-3868, 1996.

[14] F. Iori, E. Degoli, E. Luppi et al., "Doping in silicon nanocrystals: an ab initio study of the structural, electronic and optical properties," Journal of Luminescence, vol. 121, no. 2, pp. 335339, 2006. 

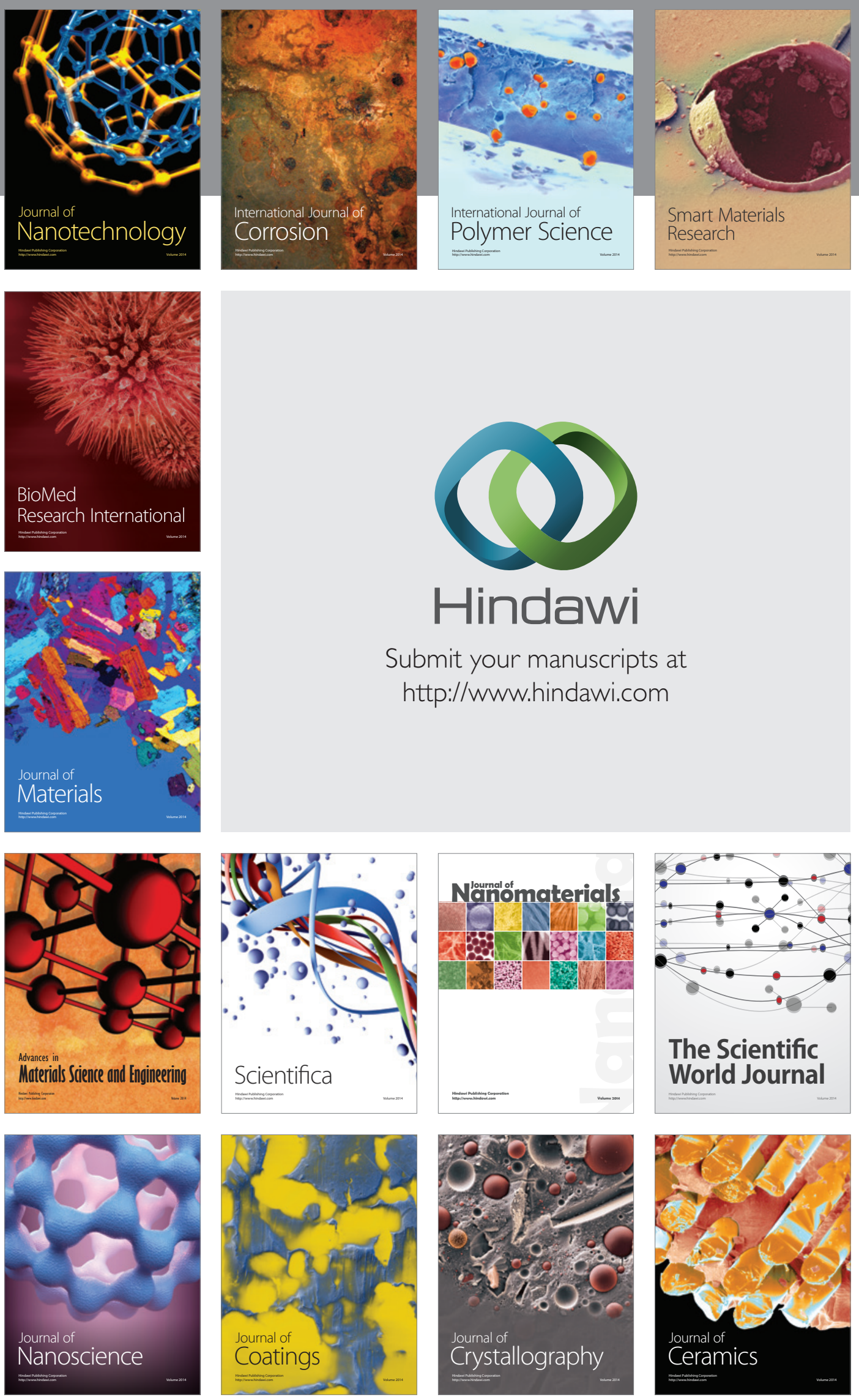

The Scientific World Journal

Submit your manuscripts at

http://www.hindawi.com

\section{World Journal}

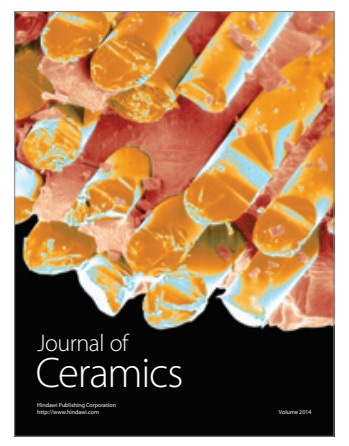

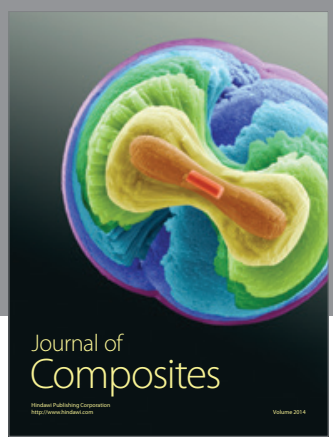
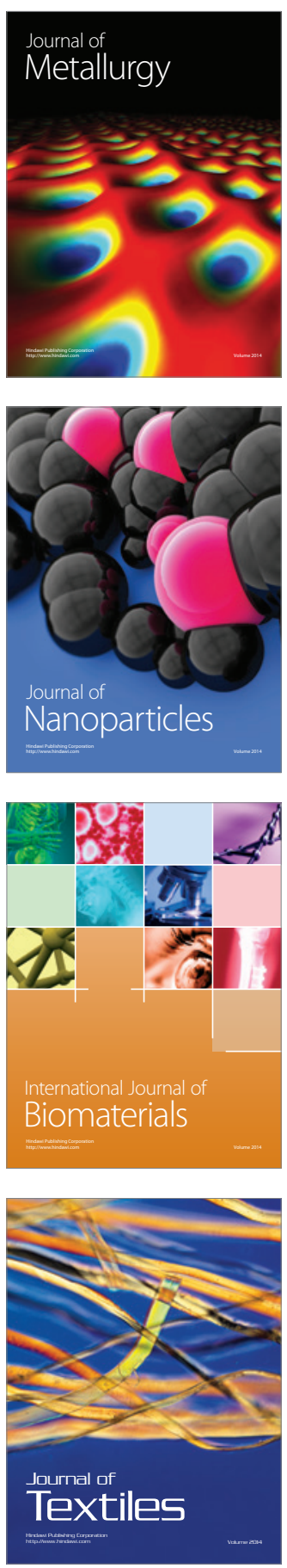